\title{
Familial spinocerebellar ataxia with skin hyperpigmentation
}

\author{
MICHAEL DARAS, ALAN J TUCHMAN, SHANTHA DAVID \\ From the Department of Neurology, New York Medical College, Lincoln Hospital, Bronx, New York, USA
}

SUMMARY Previous reports have shown the association between familial spastic paraplegia and hypopigmentation of the skin. A family is reported in which three siblings presented with progressive spastic paraparesis and cerebellar ataxia. All the siblings had large hyperpigmented naevi of the lower extremities while none of the unaffected members had a skin lesion. A definite association appears to exist between heredo-familial ataxias and disordered skin pigmentation, but the exact mechanism remains unclear.

Many syndromes with disordered skin pigmentation and abnormalities of the nervous system have been described. In two recent reports ${ }^{2}$ two families with familial spastic paraplegia were described, in which hypopigmentation of the skin was present in the affected members. We describe a family with late onset progressive spastic paraplegia with cerebellar ataxia in which the three affected members have hyperpigmented naevi in the lower extremities.

\section{Case reports}

Case 1 A 41-year-old Hispanic woman was completely normal until the age of $30 \mathrm{yr}$ when she first noticed mild weakness in her legs with impairment of balance and a tendency to trip when walking quickly. The weakness of the legs progressed and she became wheelchair-bound at the age of 36. She had no sensory loss, or bowel or bladder incontinence. However, she reported blurred vision on extreme lateral gaze and slurred speech during the past year. Her parents, who were first cousins, were neurologically normal, as were her father's five siblings and her mother's only brother. However, two of the patient's three brothers had similar neurological manifestations, which will be described below. Examination revealed the presence of a large hyperpigmented naevus on the left thigh 6 $\mathrm{cm}$ in length (fig 1) as well as several small hyperpigmented naevi on her back. She had bilateral pes cavus. There was mild dysarthria, bilateral horizontal nystagmus on lateral gaze, hypotonia and severe weakness in both lower extremities. Tendon reflexes were exaggerated at the knees and ankles. Plantar responses were bilaterally extensor. No dysmetria was noted on finger-to-nose testing, but rapid alternating movements were sluggish bilaterally. Touch,

Address for reprint requests: Michael Daras, MD, Department of Neurology, New York Medical College, Lincoln Hospital, 234 E.
149th St, Bronx, NY 10451, USA.

Received 8 July 1982. Accepted 4 March 1983 pin and temperature senses were normal but position and vibration sense were decreased in both feet. Routine laboratory investigations, including blood count and chemistry, urinalysis, serum B-12 level, VDRL, EKG, and chest, skull and complete spine radiographs were normal. Examination of the spinal fluid was also normal. Computed tomography of the head revealed bilateral cerebellar hemispheric atrophy. Motor nerve conduction velocities, sensory nerve latencies and electromyogram were normal. Skin biopsy of the hyperpigmented naevus revealed normal melanocytes.

Case 2 The patient's 45-year-old brother was normal until his early thirties when he noticed the insidious onset of progressive bilateral leg weakness. However, progression was much slower and the patient can still walk with the support of crutches. On examination he was found to have a hyperpigmented naevus on his left thigh and bilateral pes cavus. Bilateral horizontal nystagmus was noted. Muscle power was normal but both legs were spastic. Tendon reflexes were exaggerated at the knees and ankles, and plantar responses were bilaterally extensor. There was finger-to-nose and heel-to-shin dysmetria. The patient

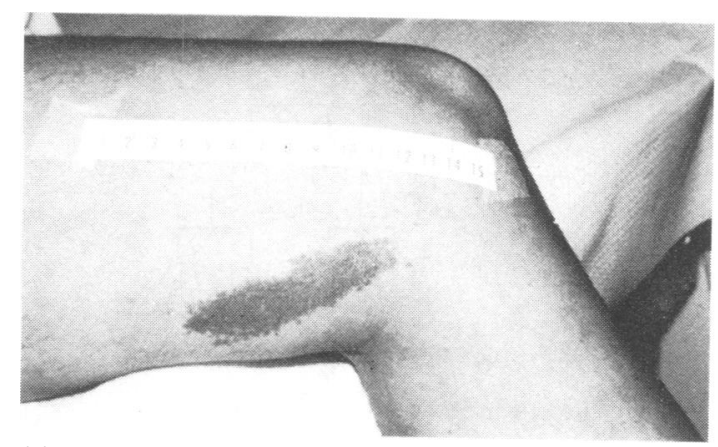

Fig 1 Photograph showing the presence of hyperpigmented naevus on the thigh in case 1. 
could walk with a spastic-ataxic gait holding on to the examiner.

Case 3 A 40-year-old brother was also normal until the age of $30 \mathrm{yr}$. His gait disturbance progressed until he became wheelchair-bound at the age of 38 . He had multiple naevi in both upper and lower extremities. His feet were flat. There was bilateral horizontal nystagmus, and moderate weakness and spasticity in both lower limbs. Tendon reflexes were exaggerated at both knees and ankles, and both plantar responses were extensor. Heel-toshin dysmetria was noted. Sensory examination revealed decreased position and vibration sense in both feet.

Other relatives The patients' parents as well as their 43-year-old brother and the four children of cases 1 and 3 were examined. All were neurologically normal and none had any skin lesions (fig 2).

\section{Discussion}

The three patients reported here have a neurological syndrome which can be classified under the broad spectrum of the spinocerebellar degenerations. They all exhibited a relatively similar neurological picture characterised by progressive paraplegia associated with exaggerated knee and ankle reflexes, bilateral Babinski sign and, in two of the patients, spasticity. They all had cerebellar involvement as shown by various degrees of dysmetria, nystagmus and dysarthria; the presence of cerebellar atrophy was shown by computed tomography in case 1 . In two of the patients (cases 1 and 3 ) there was vibration and position sense loss suggesting posterior column involvement. In addition, there was bilateral pes cavus in two patients and flat feet in the third.

Skin changes included the presence of hyperpigmented naevi in the patients but not in their healthy relatives. The expression of an almost identical syn-

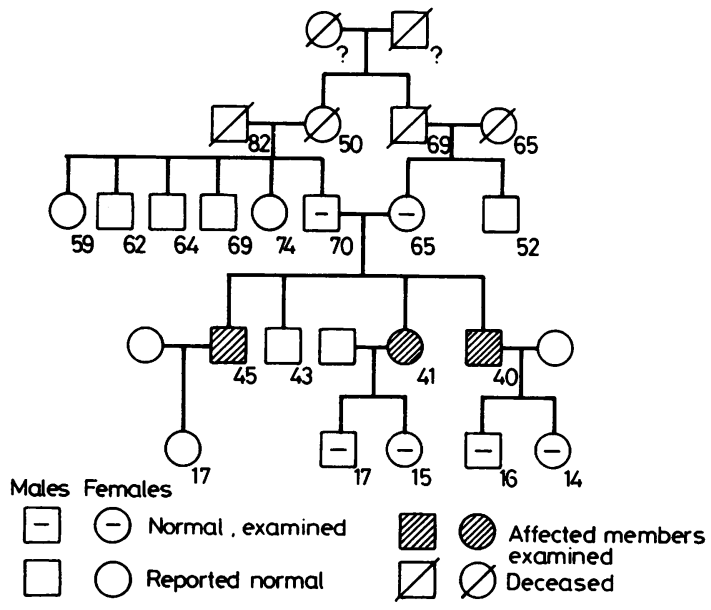

Fig 2 Pedigree of the family. drome in three siblings with unaffected consanguinous parents is consistent with an autosomal recessive inheritance.

The two previously reported families ${ }^{12}$ with progressive spastic paraplegia and disordered skin pigmentation respectively demonstrated an autosomal recessive $^{1}$ and an autosomal dominant ${ }^{2}$ mode of inheritance. The dominant neurological abnormality in these families was the presence of spastic paraparesis with peripheral nerve involvement which classifies them under the so called "complicated forms of familial spastic paraplegia". ${ }^{3}$ Our cases can also be classified under the same category as they have features consistent with a transitional form between familial spastic paraplegia and the heriditary ataxias. However, in the present cases the skin hallmark was the presence of hyperpigmented naevi, while the other two families had hypopigmentation of the skin.

Spastic paraparesis with or without cerebellar ataxia has aiso been described in other skin disorders such as xeroderma pigmentosum ${ }^{45}$ and Sjoegren-Larsson syndrome. ${ }^{6}$ Severe mental deficiency and stationary spasticity of the lower extremities similar to the one seen in cerebral palsy are characteristic of these patients. Although melanocytes may arise from neutral crest cell precursors, ${ }^{2}$ the nature of the association between neurological and dermatological abnormalities remains unclear, as in the other neurocutaneous syndromes.

\section{References}

${ }^{1}$ Abdallat A, Davis SM, Farrage J, et al. Disordered pigmentation, spastic paraparesis and peripheral neuropathy in three siblings: a new neurocutaneous syndrome: $J$ Neurol Neurosurg Psychiatry 1980;43:962-6.

${ }^{2}$ Stewart RM, Tunell G, Ehle A. Familial spastic paraplegia peroneal neuropathy and crural hypopigmentation: A new neurocutaneous syndrome. Neurology (NY) 1981;31:754-7.

${ }^{3}$ Sutherland JM. Familial spastic paraplegia. In: Vinken PJ, Bruyn GW, eds. Handbook of Clinical Neurology, vol: 22. Amsterdam North Holland Publishing Company, 1973;421-31.

${ }^{4}$ Thrush DC, Holti G, Bradley WG, et al. Neurological manifestations of xeroderma pigmentosum in two siblings. J Neurol Sci 1974;22:21-104.

${ }^{5}$ Robbins $\mathrm{JH}$, Kraemer KH, Lutzner MA, et al. Xeroderma pigmentosum. An inherited disease with sun sensitivity, multiple cutaneous neoplasms and abnormal DNA repair. Ann Intern Med 1974;80(2):221-48.

- Sjoegren T, Larsson T. Oligophrenia in conjunction with congenital ichthyosis and spastic disorders. A clinical and genetic study. Acta Psychiatr Scand 1957;Suppl 113:1. 